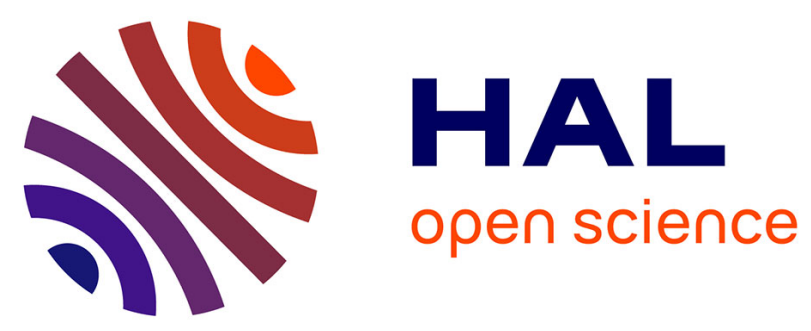

\title{
Légitimité des associations de consommateurs et vulnérabilité perçue de leurs publics : quels effets sur l'intention de confiance envers les acteurs consuméristes?
}

Marie-Christine Lichtlé, Lydiane Nabec, Dominique Roux, Corinne Chevalier

\section{To cite this version:}

Marie-Christine Lichtlé, Lydiane Nabec, Dominique Roux, Corinne Chevalier. Légitimité des associations de consommateurs et vulnérabilité perçue de leurs publics: quels effets sur l'intention de confiance envers les acteurs consuméristes?. Recherche et Applications en Marketing (French Edition), 2017, 33 (1), pp.56-77. 10.1177/0767370117738706 . hal-02022171

\section{HAL Id: hal-02022171 \\ https://hal.science/hal-02022171}

Submitted on 15 Jul 2021

HAL is a multi-disciplinary open access archive for the deposit and dissemination of scientific research documents, whether they are published or not. The documents may come from teaching and research institutions in France or abroad, or from public or private research centers.
L'archive ouverte pluridisciplinaire HAL, est destinée au dépôt et à la diffusion de documents scientifiques de niveau recherche, publiés ou non, émanant des établissements d'enseignement et de recherche français ou étrangers, des laboratoires publics ou privés. 


\title{
LÉGITIMITÉ DES ASSOCIATIONS DE CONSOMMATEURS ET VULNÉRABILITÉ PERÇUE DE LEURS PUBLICS : QUELS EFFETS SUR L'INTENTION DE CONFIANCE ENVERS LES ACTEURS CONSUMÉRISTES ?
}

\author{
Marie-Christine LICHTLE \\ Professeure des Universités, Université de Montpellier, Laboratoire MRM \\ Université de Montpellier \\ Institut Montpellier Management \\ MRM \\ Espace Richter \\ Avenue Raymond Dugrand \\ 34960 Montpellier cedex 2 \\ E-mail : Marie-Christine.Lichtle@umontpellier.fr
}

\section{Lydiane NABEC}

Maître de Conférences HDR, Université de Paris-Sud/Paris-Saclay, Laboratoire RITM

54 Boulevard Desgranges

92331 Sceaux Cedex

E-mail : lydiane.nabec@u-psud.fr

\section{Dominique ROUX}

Professeure des Universités, Université de Reims Champagne-Ardenne, Laboratoire REGARDS

Université de Reims Champagne-Ardenne

UFR des Sciences Economiques, Sociales et de Gestion, Laboratoire Regards,

Bâtiment Recherche, 57 bis rue Pierre Taittinger, 51096 Reims cedex

E-mail : dominique.roux@univ-reims.fr

\section{Corinne CHEVALIER}

Maître de Conférences, Université de Paris-Sud/Paris-Saclay, Laboratoire RITM

Université de Paris-Sud - IUT

8 Avenue Cauchy

92330 Sceaux

E-mail : corinne.chevalier@u-psud.fr

- Pour citer cet article : Lichtlé M.-C., Nabec L., Roux D. et Chevalier C. (2018), Légitimité des associations de consommateurs et vulnérabilité perçue de leurs publics : quels effets sur l'intention de confiance envers les acteurs consuméristes ?, Recherche et Applications en Marketing, 33 (1): 56-77. 


\title{
LÉGITIMITÉ DES ASSOCIATIONS DE CONSOMMATEURS ET VULNÉRABILITÉ PERÇUE DE LEURS PUBLICS : QUELS EFFETS SUR L'INTENTION DE CONFIANCE ENVERS LES ACTEURS CONSUMÉRISTES ?
}

\section{Résumé}

La confiance qu'accordent les individus aux associations de consommateurs pour protéger leurs intérêts face aux acteurs marchands est au cœur de la mission consumériste. Il est donc important d'identifier les facteurs sur lesquels repose cette confiance. Les résultats d'une étude qualitative (51 répondants) et d'une étude quantitative (315 individus) montrent que l'intention d'accorder sa confiance à une association de consommateurs dépend du degré de légitimité cognitive, pragmatique et morale qui lui est reconnu. Par ailleurs, l'effet de la légitimité pragmatique sur l'intention de confiance varie selon la vulnérabilité perçue des individus face aux acteurs marchands. En revanche, la vulnérabilité perçue ne modère pas la relation entre la légitimité cognitive ou la légitimité morale et l'intention de confiance dans les associations : elle a une influence directe sur cette variable à expliquer.

\section{Mots clés}

Associations de consommateurs, consumérisme, confiance, légitimité, vulnérabilité.

\section{CONSUMER ORGANIZATIONS' LEGITIMACY AND CONSUMER'S PERCEIVED \\ VULNERABILITY: WHAT CONSEQUENCES ON CONSUMERS' INTENT TO TRUST CONSUMER ORGANIZATIONS?}

\begin{abstract}
Trust in consumer organizations (COs), in particular for defending their interests against market practitioners, is at the heart of consumerism and consumer protection. Therefore, it is crucial to investigate on which factors they ground their willingness to rely on COs. Based on a qualitative study (51 informants) and a quantitative study (315 respondents), our study shows that their intention to trust COs depends on the way their cognitive, pragmatic and moral legitimacy is appraised. They also demonstrate that only the effect of pragmatic legitimacy on intent to trust varies according to perceived vulnerability. Vulnerability does not moderate the relationship between cognitive or moral legitimacy. Rather, vulnerability influences intent to trust directly.
\end{abstract}

\section{Keywords}

Consumer organizations, trust, legitimacy, vulnerability. 


\section{Introduction}

Les mouvements de défense des consommateurs sont aussi anciens que le commerce lui-même (Bihl-Willette, 1984 ; Fontaine, 2014), même s'ils ne se sont réellement structurés qu'après la seconde guerre mondiale. Dès 1962, le Kennedy Act aux Etats-Unis garantit à chaque citoyen quatre droits fondamentaux : la sécurité, l'information, le choix et l'écoute (Buskirk et Rothe, 1970). En France, le mouvement coopératif (Gide, 1898/1931) et la Ligue sociale d'acheteurs (Chessel, 2012, 2016) ont été créés. Les chantiers de reconstruction du Plan Marshall ont ensuite fait naître les principales instances de représentation et de défense des consommateurs (Chatriot, 2004). La plus ancienne est l'Union Fédérale de la Consommation (UFC), créée en 1951 à l'initiative du «Commissariat général à la productivité », au service duquel elle a été pensée (Pflieger, 2003). Avec pour objectif " d'informer, d'éduquer et de protéger les consommateurs contre des pratiques trompeuses, des activités anti-concurrentielles, des produits dangereux, et tout type d'abus marketing " (Webster, 1973: 90), la mission consumériste s'est développée comme une manière de représenter les consommateurs et comme un levier de dynamisation et de modernisation de l'économie d'après-guerre.

Fort de six décennies d'activités et du soutien que l'Etat lui a apporté sous la forme d'agréments, de financements et de législations ${ }^{1}$, le mouvement consumériste est donc une institution, une sorte d'« allant de soi » (Suchman, 1995: 582) que sa persistance et son bienfondé rendent apparemment peu questionnable. Pourtant, en dépit de son caractère historique et institutionnalisé, des interrogations se font jour quant à sa légitimité. Tout d'abord, la neutralité des associations de consommateurs est mise en doute du fait qu'une partie d'entre elles est idéologiquement marquée sur le plan politique ou religieux. De plus, des consommateurs craignent que les tests comparatifs qu'elles réalisent soient financés par des acteurs marchands (Nabec et al., 2015). Ensuite, le «marché de l'information consumériste » est surencombré et il est parfois difficile de savoir qui communique (Roux, Chevalier et Nabec, 2015). En conséquence, les consommateurs ont du mal à identifier quelles sources sont véritablement indépendantes. Par exemple, lorsqu'ils confondent une association de consommateurs avec une enseigne de distribution sous prétexte que cette dernière diffuse un comparateur de prix, la perception de légitimité des associations se trouve mise à mal (Nabec et al., 2015). Par ailleurs, des scandales politiques, économiques ou sanitaires font plus largement craindre aux consommateurs-citoyens que l'Etat ne soit pas en mesure de protéger

\footnotetext{
${ }^{1}$ Dans la suite du Code de la consommation issu de la loi du 26 juillet 1993, la loi récente du 17 mars 2014 , dite loi Hamon, a consacré l'existence de l'action de groupe et donné aux associations de nouveaux moyens d'action dans la défense collective des consommateurs.
} 
leurs droits (Gabaix, Landier et Thesmar, 2012). Ce climat de confusion alimente ainsi un sentiment de suspicion généralisée préjudiciable à la confiance envers les acteurs marchands (Benedicktus et al., 2010 ; Darke, Ashworth et Ritchie, 2008), mais également envers les organisations qui oeuvrent à des causes légitimes (Vestergaard, 2014).

Trois questions se posent alors, en écho aux réflexions sur l'amélioration du bien-être du consommateur (Gorge, Özçağlar-Toulouse et Toussaint, 2015) : les associations de consommateurs sont-elles encore perçues comme légitimes par leurs publics ? Si oui, et compte tenu de l'absence fréquente d'expérience directe avec ces organisations ${ }^{2}$, cette perception de légitimité influence-t-elle l'intention qu'ont ces publics de leur faire confiance ? Enfin, considérant que les individus sont inégaux dans leur capacité à se défendre (Chazal, 2000), quel rôle joue leur vulnérabilité perçue dans cette relation ? En effet, alors que les capacités des consommateurs à se défendre collectivement ont été récemment illustrées (Roux, Chevalier et Nabec, 2015), qu'en est-il de l'impuissance des individus isolés à résoudre seuls un litige (Bourdieu et Pinto, 2013 ; Roux, 2012) ? Le droit leur reconnait certes une position de faiblesse présumée vis-à-vis des acteurs marchands, du fait même qu'ils ne sont pas des professionnels (Calais-Auloy et Steinmetz, 1996). Mais il est particulièrement pertinent d'analyser si les plus vulnérables d'entre eux, selon leur perception, sont davantage susceptibles d'accorder leur confiance aux associations de consommateurs. On se demandera donc si leur sentiment de vulnérabilité modère l'effet de la légitimité perçue de ces organisations sur l'intention de leur faire confiance.

Pour répondre à ces questions, nous présentons en premier lieu le cadre conceptuel de la recherche, puis l'étude qualitative et les hypothèses qui en découlent, avant de décrire la méthodologie quantitative utilisée et les résultats obtenus. En conclusion, nous formulons des implications théoriques et managériales, et mettons en évidence les limites et les perspectives futures de cette recherche.

\footnotetext{
${ }^{2} \mathrm{C}$ 'est bien une intention que nous cherchons à capter et non la mesure directe de la confiance dans ces acteurs car seulement $5 \%$ des Français étaient adhérents, il y a dix ans, à une association de consommateurs (CREDOC, 2005: 19). En dépit d'un score de confiance élevé, cette étude souligne globalement l'érosion de l'engagement citoyen dans les associations de consommateurs. La plus importante - l'UFC Que-Choisir - ne compte aujourd'hui que 140000 adhérents, même si sa publication mensuelle «Que Choisir » est censée toucher 400 000 abonnés et 4,5 millions de lecteurs (http://www.conso.net/content/ufc-que-choisir-association-deconsommateurs).
} 


\section{Les effets de la légitimité d'une organisation sur l'intention de lui faire confiance : le cadre théorique}

\section{La légitimité d'une organisation}

Issue des sciences politiques, la légitimité se définit comme la perception, par un public donné, du caractère adéquat et approprié des actions d'une organisation à l'intérieur d'un système particulier de normes, de valeurs et de croyances (Suchman, 1995). Elle découle d'un processus de jugements et de réponses formés à partir de la nature des activités, des comportements et des valeurs de cette organisation (Bitektine, 2011). A l'échelle d'une entreprise, ce sont les actions marketing mises en oeuvre qui vont contribuer à rendre l'offre légitime, c'est-à-dire à l'intégrer dans des schémas de sens partagés (Chaney et Ben Slimane, 2014).

Deux modalités de jugement sont classiquement retenues pour appréhender ce concept (Bitektine, 2011 ; Suchman, 1995) : la légitimité cognitive et la légitimité sociopolitique.

La «légitimité cognitive », tout d'abord, repose sur des évaluations de sens commun qui font de certaines organisations une évidence, une réalité non questionnable. Il ne s'agit pas ici d'estimer le bien-fondé de leurs activités, mais de saisir le caractère immédiatement appréhendable ou compréhensible de leur présence (Suchman, 1995). En cela, les associations de consommateurs agréées sont légitimes sur le plan cognitif : elles existent depuis près de 70 ans et l'État leur délivre un agrément de défense des consommateurs (Laurent, 2009). Il leur permet ainsi d'obtenir ce que Meier et Scheier (2008) appellent une légitimité élective. Toutefois, si elles cessaient d'être reconnues par des publics qui ne les identifiaient plus (Roux, Chevalier et Nabec, 2015), leur légitimité cognitive pourrait se trouver compromise.

La «légitimité sociopolitique », ensuite, porte sur l'évaluation par les individus des bénéfices que présentent certaines organisations à l'aune de leurs normes et de leurs valeurs (Suchman, 1995). En cela, et toutes choses égales par ailleurs, cette forme de légitimité est fortement dépendante de leurs croyances, attitudes, valeurs ou expériences vécues.

Selon Bitektine (2011), les jugements de légitimité sociopolitique se divisent eux-mêmes en deux. En premier lieu, les jugements egotropiques (ou légitimité pragmatique) sont formés à partir de l'évaluation de l'efficacité et de la capacité des organisations à satisfaire leurs publics (Suchman, 1995). Appliquée aux associations de consommateurs, la légitimité egotropique s'apprécie au travers des moyens qu'elles peuvent déployer pour informer et défendre les consommateurs (Laurent, 2009). Quand il s'agit de les aider à résoudre leurs litiges, Roux, Chevalier et Nabec (2015) montrent en particulier qu'elles doivent être facilement identifiables et accessibles pour que les consommateurs les sollicitent, notamment en cas de 
litige. En plus de cette accessibilité, une question plus large se pose aujourd'hui quant à leur capacité à résoudre des problèmes sur le plan opérationnel et juridique. Sur le premier point en effet, leur efficacité dépend largement des moyens financiers dont elles disposent pour mener à bien leurs missions. Or, ceux-ci vont en se raréfiant, ce qui réduit d'autant leur capacité « à recruter et à rémunérer des professionnels » (Laurent, 2009: 14). Sur le second point, la loi sur la Consommation, dite loi Hamon, leur permet désormais d'introduire l'action de groupe et d'agir en défense d'une somme d'intérêts individuels. Toutefois, conférer à l'association de consommateurs ce droit élargi leur donne également « une véritable responsabilité », car en l'absence de ressources supplémentaires, le consommateur pourrait «faire des reproches à l'organisation, notamment en cas d'inaction » (Azar-Baud, 2016: 198).

En second lieu, les jugements sociotropiques (ou légitimité morale) portent sur les bénéfices que les organisations sont susceptibles de procurer à la société tout entière (Suchman, 1995). Ils reposent donc avant tout sur la valeur sociétale accordée à la mission consumériste. Or, l'émiettement du paysage consumériste renforce le manque de visibilité des associations. Leur nombre et l'hétérogénéité de leurs sensibilités entraînent également une possible confusion entre certains intérêts catégoriels - portés par les associations d'origine syndicale notamment et la défense des consommateurs qui doit être exercée «à titre prépondérant » (Laurent, 2009: 28).

En résumé, la légitimité des associations de consommateurs découle des jugements qui sont portés sur leur ancrage institutionnel (légitimité cognitive), sur leur capacité perçue à se faire connaître, à se rendre accessible et à protéger les consommateurs (légitimité pragmatique) et sur leur contribution au bien-être des individus, notamment les moins aptes à s'informer et à se défendre (légitimité morale). Nous définissons donc la légitimité des associations de consommateurs comme «leur capacité à être identifiées comme des acteurs naturels de la protection des consommateurs, à déployer les moyens adéquats de leur protection, et à veiller collectivement à ces missions auprès des plus fragiles ».

Le concept de légitimité demeure spécifique en dépit de sa proximité avec les notions de célébrité, de statut ou de réputation. La célébrité, tout d'abord, se définit par le fait d'attirer l'attention d'une audience et de susciter chez elle des affects positifs. Cette performance, en soi, n'est pas discriminante pour établir la légitimité d'une organisation (Rindova, Pollock et Hayward, 2006). De même, le statut et la réputation n'empruntent pas aux mêmes fondements théoriques - l'institutionnalisme - que la légitimité. Le statut est, au sein de la théorie des réseaux, une mesure des qualités évaluatives de la position d'un acteur par rapport aux autres. Quant à la réputation, elle relève de la théorie du signal dans laquelle des indicateurs de 
performance attestent des capacités d'une organisation à produire de la valeur. Ni le statut, ni la réputation ne sont donc fondés sur des critères politiques d'acceptation et de pertinence sociale, contrairement à la légitimité (Bitektine, 2011).

La confiance accordée à une organisation et le rôle de la légitimité dans la formation de l'intention de confiance

La confiance se définit à la fois comme un état psychologique fait de croyances, d'attentes et de présomptions « accumulées quant à la crédibilité, à l'intégrité et la bienveillance » du partenaire de l'échange, en amont de son comportement (Gurviez et Korchia, 2002: 47). En aval, c'est une composante conative manifestée par une intention (de faire confiance) ou par un comportement (confiant) (Chouk et Perrien, 2003 ; Guibert, 1999). La confiance, en tant qu'état psychologique, est ainsi fondée sur des déterminants d'ordre cognitif (comme la crédibilité et l'intégrité) et affectifs (comme la bienveillance). Ils conduisent les acteurs à se prêter mutuellement des qualités de compétence, d'honnêteté et de volonté d'agir pour le bien et le bonheur de l'autre (Morgan et Hunt, 1994). De la même manière, les travaux sur la confiance institutionnelle tissée entre un individu et une personne morale - commerçants, fabricants, Etat, associations de consommateurs - la décrivent «comme la volonté délibérée de compter sur la fiabilité et la bienveillance d'une partie en qui l'on croit» (Benamour, 2000: 128).

Dans sa dimension conative, la confiance traduit l'intention ou la volonté d'une des parties de s'en remettre à l'autre (Moorman, Zaltman et Deshpande, 1992; Rotter, 1971). Cette confiance peut être nourrie par le résultat positif de relations professionnelles antérieures (Bidault et Jarillo, 1995 ; Guibert, 1999 ; Mayer, Davis et Schoorman, 1995) ou par la qualité des expériences passées avec une organisation (Bryce, 2007). En effet, les partenaires ou les publics de cette dernière ont alors une meilleure connaissance de ses actions, et celle-ci nourrit en retour leur intention de confiance.

Dans le domaine de la consommation, les recherches ont montré le rôle médiateur de la confiance dans la relation entre la satisfaction cumulée et l'engagement, qui influence à son tour la fidélité (Aurier, Benavent et N'Goala, 2001 ; Lichtlé et Plichon, 2008 ; Cissé-Depardon et N'Goala, 2009 ; Gustafsson, Johnson et Roos, 2005 ; Morgan et Hunt, 1994 ; Sirieix et Dubois, 1999). Toutefois, se fondant sur la satisfaction des consommateurs, ces recherches présupposent nécessairement l'existence d'une expérience préalable. Qu'en est-il lorsque l'expérience n'a pas lieu ? S'il est impossible, dans ce cas, d'appréhender la satisfaction et donc la confiance sur une base ex-post (Gurviez et Korchia, 2002), il est possible, en revanche, d'anticiper les intentions d'engagement dans la relation par des variables autres que la 
satisfaction (Bartikowsky, Chandon et Müller, 2007). En effet, présumer des compétences, de l'honnêteté, et de la capacité d'un partenaire à prendre en compte l'intérêt d'autrui, c'est-à-dire lui faire confiance a priori, peut s'appuyer sur d'autres éléments de connaissance disponibles. C'est ce que montrent les recherches qui ne mesurent pas directement la confiance des individus dans les organisations, mais qui l'appréhendent au travers de leur légitimité perçue (Arnold, Handelman et Tigert, 1996; Handelman et Arnold, 1999). Ainsi, plus les organisations sont fortement perçues comme légitimes, plus les consommateurs manifestent des intentions comportementales favorables à leur égard (une recommandation, une attitude favorable à l'implantation d'un nouveau magasin, une faible intention de boycott ou encore une répétition de la fréquentation).

Dans le champ institutionnel qui nous concerne ici, Eichholtzer (2010) s'intéresse également au lien entre légitimité et confiance. Il montre que la légitimité perçue de l'Union Européenne est fondamentale pour obtenir la confiance et l'adhésion des citoyens. A l'appui de cette théorisation du lien légitimité-confiance, on retiendra le caractère anticipatoire de la confiance. Celle-ci agit, en effet, comme une volonté ou une intention fondée sur des espérances, des croyances en la crédibilité et en l'intégrité du partenaire, ainsi que sur des présomptions de sa bienveillance (Moorman, Zaltman et Deshpandé, 1992). Considérant que ces contributions sont applicables aux associations de consommateurs, nous suggérons que la légitimité perçue d'une association constitue, en l'absence d'expérience préalable, un déterminant de l'intention de lui faire confiance.

Pour qu'un individu accorde sa confiance à une organisation, il faut toutefois tenir compte de sa vulnérabilité et de l'incertitude de la situation (Deutsch, 1962 ; Mayer, Davis et Schoorman, 1995 ; Smith et Barclay, 1997). Selon certains chercheurs, la vulnérabilité serait même consubstantielle à la confiance, du fait que l'un des partenaires se trouve généralement en situation de dépendance par rapport à l'autre (Bartikowsky, Chandon et Müller, 2007 ; Mayer, Davis et Schoorman, 1995). Si cette approche a surtout été développée dans le cadre des relations entreprise-consommateur, dans lesquelles l'asymétrie joue en défaveur du second, nous examinons ici la vulnérabilité perçue du consommateur dans le cadre de la confiance qu'il accorde aux associations oeuvrant pour sa défense.

\section{Le rôle de la vulnérabilité perçue du consommateur}

La vulnérabilité est un concept parfois assimilé à tort à la discrimination, la stigmatisation, les situations de désavantage découlant de certaines caractéristiques individuelles, biologiques (âge, appartenance ethnique, handicap), psychosociales (statut économique, niveau 
d'éducation, illettrisme, problèmes d'apparence) ou d'état (deuil, fragilités temporaires liées à une situation) (Baker, Gentry et Rittenburg, 2005).

En réalité, la vulnérabilité n'est pas une notion attachée par principe à une catégorie d'individus désignés par d'autres comme vulnérables («perceived vulnerability») (Smith et Cooper-Martin, 1997). Elle doit, au contraire, être envisagée en tant que sentiment ressenti par celui ou celle qui se perçoit comme réellement vulnérable («actual vulnerability»). Plus encore, la vulnérabilité résulte d'une interaction entre un individu et une situation. Dans notre cas, c'est la situation de consommation qui peut provoquer cet «état d'impuissance lié à un déséquilibre dans les interactions marchandes ou à l'exposition à des messages marketing et à des produits» (Baker, Gentry et Rittenburg, 2005: 134). Elle correspond, pour le consommateur, au fait de se sentir dépourvu de contrôle «sur un contexte qui entrave l'atteinte de ses buts et dont l'expérience affecte la perception personnelle et sociale qu'il a de lui-même » (Baker, Gentry et Rittenburg, 2005: 134). Ce manque de contrôle le rend alors «dépendant de facteurs externes dont il doit attendre qu'ils créent de la justice sur le marché »(Baker, Gentry et Rittenburg, 2005: 134). Certes, l'individu peut présenter des formes de fragilité liées à un manque de compétences physiques (handicap), à des sensibilités particulières (allergies), à des difficultés mentales ou des faiblesses cognitives (naïveté). Mais la vulnérabilité découle également de la manière dont des facteurs situationnels particuliers, notamment les environnements commerciaux au sens large, sont susceptibles d'exacerber les dangers auxquels il se trouve confronté (Morgan, Schuler et Stoltman, 1995).

$\mathrm{Si}$ on considère que remédier aux problèmes d'asymétrie entre professionnels et profanes fait partie du rôle dévolu aux associations de consommateurs (Chazal, 2000), la vulnérabilité entretient donc une forte proximité avec les actions de protection du consommateur et son bien-être (Gorge, Özçağlar-Toulouse et Toussaint, 2015). Baker, Gentry et Rittenburg (2005) indiquent, toutefois, que vulnérabilité et besoin de protection ne sont pas des notions interchangeables. En effet, si les membres d'un groupe sous protection sont susceptibles de se sentir vulnérables - le consommateur étant l'un d'entre eux -, le fait d'appartenir à ce groupe n'implique pas que chaque individu se juge fragile. Certains auteurs (Martin, 1987 ; SinayCytermann, 1994) se sont, en effet, attachés à montrer qu'il existait des consommateurs «abusifs », largement surprotégés dans leurs rapports avec des professionnels. Par ailleurs, des individus peuvent posséder un niveau d'expertise et de compétence au moins égal à celui de leur cocontractant (par exemple, un banquier qui demande un prêt ; un informaticien qui achète un ordinateur...). De fait, si le législateur a privilégié une vision générale, équitable et 
abstraite de la vulnérabilité des consommateurs, la manière dont ces derniers perçoivent leur propre vulnérabilité peut être variable.

En conséquence, la variabilité de ce sentiment individuel rend pertinent l'examen de sa relation avec l'intention d'avoir confiance dans les associations de consommateurs. Afin de préciser la nature de ce lien, une étude qualitative a été conduite.

\section{L'étude qualitative et les hypothèses de la recherche}

\section{L'étude qualitative : méthodologie et résultats}

L'étude qualitative avait pour objectif d'examiner les facteurs susceptibles de conduire les individus à accorder leur confiance aux associations de consommateurs. En raison de la variété des incidents critiques recueillis dans le discours des répondants, 51 entretiens individuels ont au total été conduits. Ce nombre élevé a permis de s'assurer «qu'il n'existe plus d'information supplémentaire capable d'enrichir la théorie » (Thiétart, 2003: 216). Les répondants, recrutés selon la méthode «boule de neige », avaient tous pour point commun d'avoir rencontré un problème avec un professionnel (un fabricant, un distributeur, un prestataire de service...). Dans les démarches relatées, le fait d'avoir contacté (ou non) une association de consommateurs était particulièrement examiné. Le guide d'entretien comportait trois grandes parties : 1/ la narration du problème, des actions entreprises et des acteurs consultés ; 2/ la connaissance des associations de consommateurs, de leurs missions et les représentations attachées à leur action ; 3/ les attentes plus générales en matière d'information et de défense. Il s'agissait d'évaluer le degré de connaissance des associations de consommateurs par les répondants et de comprendre la manière dont ils les percevaient, selon leurs besoins et leurs profils. L'échantillon était composé d'individus aux caractéristiques sociodémographiques variées, qui différaient également quant à leur connaissance et à leur fréquentation des associations de consommateurs (annexe 1). Les entretiens ont été enregistrés et intégralement retranscrits. Une analyse de contenu thématique a été réalisée afin de faire émerger les thèmes et sous-thèmes des discours. Les auteurs ont procédé au codage séparé des dix premiers entretiens, puis discuté ensemble de leur grille d'analyse avant de la mettre en commun. Cette grille a été enrichie au fur et à mesure de la collecte. Elle fait ressortir l'importance de la légitimité perçue des associations de consommateurs dans l'intention de leur faire confiance ${ }^{3}$ : . La légitimité cognitive ressort au travers de l'histoire, des réalisations et de la continuité des associations de consommateurs. Ainsi pour Aline (55 ans), le fait « d'exister depuis très

\footnotetext{
${ }^{3}$ Seuls les éléments relatifs au rôle de la légitimité et de la vulnérabilité dans l'intention de confiance sont présentés, l'étude ayant poursuivi des objectifs complémentaires qui n'ont pas été détaillés dans cet article.
} 
longtemps » est un gage de confiance, ce que confirme Béatrice (44 ans) : «J'ai confiance car je les connais de par leur passé historique ». Nathalie (44 ans) se réfère quant à elle au caractère de prédictibilité de leurs activités : "J'ai confiance car elles ont prouvé par le passé qu'elles pouvaient faire réagir des secteurs ». Le caractère institutionnel du consumérisme, dans une continuité historique, est également souligné par Victor (60 ans) : «C'est pour ça que je pense qu'on peut avoir confiance en elles, c'est parce que maintenant, elles sont bien installées ». Finalement, ce caractère d' « allant de soi » est résumé par Alice (34 ans) : «Elles sont toujours là sur le créneau pour les grands problèmes, elles sont toujours là quand il y a des soucis ». Ces différents verbatim illustrent l'influence positive de la légitimité cognitive sur l'intention de confiance envers les associations.

. La légitimité pragmatique des associations est appréciée selon leur efficacité et leur capacité à répondre aux besoins de leurs publics. Comme l'indique Thierry (26 ans) : " J'ai vraiment confiance en ces associations de consommateurs, qui m'ont apporté une grande aide dans mes choix de produits ». Plus généralement, Mireille (50 ans) affirme : «J'ai confiance parce que dans tout ce que j'ai vu, entendu et lu, je m'aperçois que, par leur intermédiaire, beaucoup de consommateurs réussissent à gagner ou avoir gain de cause ». C'est en particulier sur la base de leurs compétences et de leur expertise que Monique (59 ans) pense qu'elles sont dignes de foi et efficaces : «Je les crois tout à fait crédibles. Elles ont des gens très spécialisés dans chaque domaine » Ainsi, il ressort que la légitimité pragmatique, fondée sur le savoir-faire et les résultats obtenus par les associations de consommateurs, exerce une influence positive sur l'intention de leur faire confiance.

. La légitimité morale des associations correspond, enfin, au caractère socialement désirable de leur action. Elle est fortement associée à leur distance perçue avec le monde marchand, au caractère bénévole de leurs activités et à leur indépendance financière, ce que souligne Aurore (20 ans) : «L'image des associations de consommateurs, c'est crédible parce que c'est à but non lucratif. Donc, s'il n'y a pas d'argent en jeu, normalement, c'est un peu plus impartial ». De même, Cécile F. (52 ans) indique : «Je leur fais confiance car j'ai l'impression qu'elles ne font pas de business, qu'elles n'aident pas les entreprises à faire du chiffre d'affaires ». Une autre répondante (Cécile D., 48 ans) considère également «qu'elles sont fiables surtout parce qu'elles ne sont intéressées par rien d'autre que l'intérêt des consommateurs». L'intention de confiance est alors positivement influencée par la légitimité morale des associations.

Les résultats éclairent, en dernier lieu, le rôle de la vulnérabilité perçue dans l'intention des répondants de faire confiance aux associations de consommateurs. Antoine (52 ans) indique 
ainsi que leur mission consiste précisément à « défendre les gens qui n'ont pas la capacité ou le temps de se défendre » par exemple "quand tu as un litige où là, tu te sens un peu démuni». De même Christine (53 ans) confirme que s'adresser à une association, " c'est défendre le consommateur qui est démuni face à la grande distribution ». Ces extraits laissent donc supposer un effet direct de la vulnérabilité perçue des consommateurs sur la confiance qu'ils pourraient accorder aux associations. Toutefois, les discours conduisent aussi à envisager que le degré de vulnérabilité du consommateur pourrait être un modérateur de la relation entre la légitimité perçue des associations et l'intention de leur faire confiance. C'est ce qu'illustre Monique (59 ans) : «Elles ont des avocats, ça peut même aller très loin, hein. Elles peuvent résoudre des litiges en justice. Ça peut aller très loin, les démarches! Elles font un gros boulot qu'on ignore, hein! J'ai pas mal de petits problèmes à résoudre et j'irai peutêtre les voir, justement.... J'ai peur de me faire avoir, j'ai peur de dépenser à tort, je n'aime pas tomber sur des produits de mauvaise qualité par exemple ». Cet extrait montre que la légitimité pragmatique des associations (les moyens qu'elles peuvent déployer pour la défendre) influence l'intention de confiance que cette répondante place en elles (en envisageant de les contacter), en raison de son fort degré de vulnérabilité perçue (la peur de se « faire avoir »).

\section{Les hypothèses de la recherche}

En nous appuyant sur l'ensemble des travaux évoqués ci-dessus et sur les résultats de l'étude qualitative, nous postulons que la vulnérabilité perçue du consommateur influence positivement son intention de faire confiance aux associations de consommateurs. Aussi, nous posons l'hypothèse suivante :

\section{H1. Plus le consommateur se perçoit comme vulnérable face aux acteurs marchands, plus il a l'intention de faire confiance aux associations de consommateurs.}

Ensuite, considérant que l'intention de soutenir une organisation est d'autant plus grande qu'elle est perçue comme légitime (Eichholtzer 2010 ; Handelman et Arnold, 1999), nous postulons un effet positif de la légitimité perçue des associations de consommateurs sur l'intention de leur faire confiance. Par ailleurs, compte tenu des développements précédents sur le rôle de la vulnérabilité perçue du consommateur face aux acteurs marchands, nous supposons que la relation entre la légitimité perçue des associations et l'intention de leur faire confiance est d'autant plus forte que l'individu se sent vulnérable. En effet, plus celui-ci se 
sent incapable de protéger ses intérêts par lui-même, plus l'intention de faire confiance à des acteurs qu'il considère comme légitimement missionnés pour cet objectif, devrait être forte. La légitimité perçue des associations reposant sur trois facettes - cognitive, pragmatique et morale -, nous posons donc les hypothèses suivantes :

H2a. Plus la légitimité cognitive des associations de consommateurs est perçue comme forte par le consommateur, plus l'intention de leur faire confiance est forte.

H2b. La relation entre la légitimité cognitive des associations de consommateurs et l'intention de leur faire confiance est d'autant plus forte que les consommateurs se sentent vulnérables face aux acteurs marchands.

H3a. Plus la légitimité pragmatique des associations de consommateurs est perçue comme forte par le consommateur, plus l'intention de leur faire confiance est forte.

H3b. La relation entre la légitimité pragmatique des associations de consommateurs et l'intention de leur faire confiance est d'autant plus forte que les consommateurs se sentent vulnérables face aux acteurs marchands.

H4a. Plus la légitimité morale d'une association de consommateurs est perçue comme forte par le consommateur, plus l'intention de leur faire confiance est forte.

H4b. La relation entre la légitimité morale des associations de consommateurs et l'intention de leur faire confiance est d'autant plus forte que les consommateurs se sentent vulnérables face aux acteurs marchands.

\section{Figure 1 à insérer ici}

Afin de tester les hypothèses de la recherche, une étude quantitative a été réalisée en ligne.

\section{Méthodologie de la recherche}

Le contexte de l'étude : les associations de consommateurs agréées

Seules les associations de consommateurs agréées ${ }^{4}$ ont été prises en compte dans cette recherche, pour trois raisons : les associations non-agréées ne sont pas toutes engagées de

\footnotetext{
${ }^{4}$ L'agrément des associations par l'Etat suppose qu'elles répondent à trois critères (www.conso.net) : un critère d'activité (avoir une année d'existence et justifier d'une activité effective et publique en vue de la défense des
} 
manière «prépondérante » dans la défense des consommateurs (Laurent, 2009) ; elles ont souvent un faible nombre d'adhérents, sont peu connues et peu représentatives ; elles ne satisfont pas nécessairement au critère d'indépendance qui est déterminant dans la perception de légitimité des acteurs consuméristes (Nabec et al., 2015).

\section{Le recueil des données quantitatives}

Une étude quantitative a été réalisée à partir de questionnaires diffusés en ligne auprès d'un échantillon initial de 751 individus. Ensuite, seuls les répondants capables de citer un exemple correct d'associations de consommateurs exerçant une activité en France ont été retenus. Cette sélection a été réalisée à partir de la question filtre suivante: «Quelles associations de consommateurs connaissez-vous?». L'échantillon final comprend 315 individus, présentant des profils variés en termes de genre (48\% d'hommes et $52 \%$ de femmes), d'âge ( $25 \%$ de 15 à 34 ans, $27 \%$ de 35 à 49 ans et $48 \%$ de plus de 50 ans) et de profession (33\% de PCS+, 28 $\%$ de PCS- et $39 \%$ d'inactifs).

Afin de vérifier que les associations de consommateurs pouvaient rencontrer un problème de légitimité et qu'il existait une variance pour cette variable, des statistiques descriptives (notamment les moyennes et écart-types) ont été calculées pour chacune des dimensions et montrent l'existence d'une variance : la légitimité cognitive (moyenne $=4,32$; écart-type $=$ 1,22 ), la légitimité pragmatique (moyenne $=5,04$; écart-type $=0,93$ ) et la légitimité morale (moyenne $=5,14 ;$ écart-type $=1,25)$.

\section{L'opérationnalisation des variables et la mesure des construits}

Les échelles de mesure de la légitimité et de l'intention de confiance ont été adaptées à partir d'outils existants dans la littérature (Capelli et Sabadie, 2005). L'échelle de vulnérabilité perçue a été construite à partir des verbatim recueillis en phase qualitative. Il s'agit d'échelles de Likert ou sémantiques différentielles en sept points $(1=$ pas du tout d'accord et $7=$ tout à fait d'accord). Pour chacune d'entre elles, et après avoir vérifié que les données étaient factorisables (indice KMO et test de Bartlett), des analyses factorielles exploratoires ont été réalisées. Pour vérifier la structure obtenue, des analyses factorielles confirmatoires fondées 
sur un modèle d'estimation selon les Moindres Carrés Partiels ${ }^{5}$ (PLS : Partial Least Square) (Tenenhaus et al., 2005) ont été menées ensuite. Compte tenu du fait que les variables ne suivaient pas tout à fait une loi normale, une procédure de Bootstrap de 250 réplications a été mise en œuvre avec le modèle PLS Path Modeling.

\section{La légitimité perçue d'une association de consommateurs}

La légitimité perçue a été mesurée en adaptant l'échelle de Capelli et Sabadie (2005). Développée dans le contexte de la légitimité d'un annonceur publicitaire, elle présente les mêmes facettes que celles identifiées en phase qualitative et décrites par la littérature (Bitektine, 2011; Suchman, 1995). L'échelle comporte trois dimensions (tableau 1) : la légitimité cognitive (découlant de la continuité d'action des associations de consommateurs dans le temps, qui rend leur existence non questionnable); la légitimité pragmatique (liée à la capacité des associations de consommateurs à répondre efficacement à leurs publics, à être facilement identifiables et accessibles pour les aider) ; et, enfin, la légitimité morale (fondée sur la valeur sociétale de leur mission).

\section{L'intention de faire confiance à une association de consommateurs}

La dimension conative de la confiance («Trusting Intention ») a été mesurée à l'aide de l'échelle de McKnight, Kacmar et Choudhury (2004) traduite par Bartikowsky, Chandon et Müller (2007). Elle comprend traditionnellement quatre sous-dimensions : 1'acceptation de la dépendance, la volonté de suivre les conseils, la disposition à donner des informations personnelles et l'intention d'achat. L'échelle a été adaptée au cas des associations de consommateurs et réduite à la suite des analyses statistiques effectuées. Elle dispose d'une structure unidimensionnelle en cinq items (tableau 1) et reflète le fait de pouvoir compter sur ces associations et la volonté de suivre les conseils donnés.

\section{La vulnérabilité perçue du consommateur face aux acteurs marchands}

Deux catégories d'échelles permettent de mesurer la vulnérabilité du consommateur. Les premières captent l'état de faiblesse perçue de certaines catégories de populations comme les enfants ou les seniors. Les secondes, qui correspondent mieux à cette recherche, captent la

\footnotetext{
${ }^{5}$ De nombreuses raisons peuvent justifier l'utilisation de PLS (Chin et Newsted, 1999 ; Fernandez, 2012), en particulier le caractère nouveau du phénomène étudié et l'absence de respect de la condition de normalité des variables.
} 
vulnérabilité perçue par les répondants eux-mêmes, et non par des observateurs extérieurs. De telles échelles ont été utilisées dans les travaux en marketing social, pour mesurer par exemple la fragilité suscitée par les messages d'appel à la peur dans certaines campagnes de prévention contre les risques tabagiques ou l'anorexie (Janz et Becker, 1984 ; Maillard-Wilhelm, 2014 ; Werle et al., 2010). Toutefois, ces mesures sont mono-item et peu transposables à notre contexte. Face à l'absence d'outil de mesure réellement satisfaisant, nous avons construit une échelle ad hoc en cinq items à partir des verbatim issus de l'étude qualitative (tableau 1). En suivant la procédure COARSE (Rossiter, 2002), nous avons défini l'objet focal de la mesure comme étant la vulnérabilité du consommateur telle qu'il la perçoit. Nous avons considéré qu'il était lui-même l'entité évaluatrice de son état. Le fait «d'être consommateur » spécifie ensuite les dimensions du jugement, et les composantes de sa mesure.

\section{Tableau 1 à insérer ici}

\section{L'évaluation de la qualité psychométrique des échelles}

Les analyses factorielles confirmatoires ont permis de valider les structures factorielles proposées, conformes aux analyses factorielles exploratoires. Les poids factoriels (loadings) sont tous supérieurs à 0,8 (ou proches de ce seuil) et peuvent donc être considérés comme satisfaisants (Fornell et Larker, 1981) (tableau 1). L'Alpha de Cronbach et le Rhô de DillonGoldstein ont ensuite permis d'évaluer la fiabilité des outils de mesure. Les coefficients obtenus sont tous supérieurs à 0,7 conformément au seuil d'acceptabilité de Nunnally (1978). La validité convergente a été vérifiée par le fait que le poids factoriel de chaque indicateur est significatif $(t>1,96)$ et supérieur ou égal à 0,70 (Anderson et Gerbing, 1988). Un test de la variance extraite moyenne (AVE) donne une indication complémentaire sur la validité convergente. Elle doit être supérieure ou égale à 0,50 selon l'approche de Fornell et Larcker (1981) et se révèle satisfaisante pour toutes les échelles (tableau 1). Enfin, la validité discriminante a été vérifiée : la validité convergente des construits est supérieure à la corrélation au carré entre les construits (Fornell et Larker, 1981). Les résultats montrent donc que toutes les échelles sont fiables et valides (tableaux 1 et 2).

Tableau 2 à insérer ici

\section{Les résultats}

Les hypothèses ont été testées par des analyses de régressions effectuées avec le logiciel SPSS 22.0, selon les préconisations de Preacher et Hayes $(2004,2008)$ avec le modèle 1 de la 
procédure de Hayes (2012), et une procédure Bootstrap de 5000 réplications. Cette approche, préconisée par Cadario et Parguel (2014), permet l'analyse des effets modérateurs sans discrétiser a priori les variables modératrices étudiées. L'effet de chaque dimension de la légitimité des associations - cognitive, pragmatique ou morale -, de la vulnérabilité perçue des individus et de leur interaction sur l'intention de confiance a été analysé par un modèle de régression distinct. Au total, trois modèles ont été conçus (Modèle 1 : effet de la légitimité cognitive ; Modèle 2 : effet de la légitimité pragmatique ; Modèle 3 : effet de la légitimité morale) (Tableaux 3, 4 et 5). Dans chacun d'eux, les effets des deux dimensions restantes de la légitimité ont été contrôlés en les introduisant en tant que covariables. Les effets d'interaction ont, enfin, été représentés graphiquement à l'aide de la procédure de Aiken et West (1991) (Figures 2, 3 et 4). Les variables utilisées ont été calculées à partir des moyennes des scores obtenus par les items composant chaque dimension.

\section{Effet direct de la vulnérabilité perçue sur l'intention de confiance}

L'effet direct de la vulnérabilité perçue des individus sur leur intention d'accorder leur confiance aux associations de consommateurs (H1) a été testé dans chacun des trois modèles de régression (Modèle $1: \beta=0,11 ; p<0,01$; Modèle $2: \beta=0,13 ; p<0,01$; Modèle $3: \beta=$ $0,11 ; p<0,001)$. Il est significativement positif (H1 est validée). Ainsi, plus les individus se sentent vulnérables face aux acteurs marchands, plus ils ont l'intention d'accorder leur confiance aux associations de consommateurs. Inversement, moins ils se perçoivent vulnérables, plus faible est leur intention de confiance à leur égard (tableaux 3 à 5).

Effets de la légitimité cognitive et de la vulnérabilité perçue sur l'intention de faire confiance aux associations de consommateurs

Les effets de la légitimité cognitive des associations de consommateurs, de la vulnérabilité perçue des individus et de leur interaction sur leur intention de leur accorder leur confiance (H2) ont été analysés dans le premier modèle de régression (Modèle $1: \mathrm{R}^{2}=42 \% ; F=$ 45,50***) (Tableau 3 ; Figure 2). Les résultats montrent un effet direct significatif de la légitimité cognitive perçue des associations de consommateurs sur l'intention de leur faire confiance $(\beta=0,13 ; p<0,05)$ (H2a est validée). Les individus ont donc d'autant plus l'intention d'accorder leur confiance aux associations de consommateurs qu'ils jugent que leur présence dans le paysage institutionnel «va de soi » et se situe dans la continuité de leur existence. Néanmoins, l'interaction de la perception de légitimité cognitive des associations de consommateurs avec la vulnérabilité perçue des individus n'est pas significative $(\beta=0,02 ; p$ 
$=0,43$ ) (Figure 2), contrairement à ce qui était attendu (Hypothèse H2b). Autrement dit, elle explique l'intention des individus de leur faire confiance, quel que soit le niveau de vulnérabilité perçue de ces derniers face aux acteurs marchands (H2b n'est pas validée).

\section{Tableau 3 et Figure 2 à insérer ici}

Effets de la légitimité pragmatique et de la vulnérabilité perçue sur l'intention de faire confiance aux associations de consommateurs

Les effets de la légitimité pragmatique des associations de consommateurs, de la vulnérabilité perçue des individus et de leur interaction sur leur intention de leur accorder leur confiance (H3) ont été analysés par le deuxième modèle de régression (Modèle $2: \mathrm{R}^{2}=43 \% ; F=$ 47,32***) (Tableau 4 ; Figure 3). La légitimité pragmatique perçue des associations de consommateurs influence positivement et de manière significative l'intention de confiance des individus $(\beta=0,59 ; \mathrm{p}<0,01)$ (H3a validée). Autrement dit, plus ils jugent que les associations de consommateurs sont capables de les aider et de répondre efficacement à leurs problèmes, plus ils ont l'intention de leur faire confiance. Inversement, lorsqu'ils les perçoivent peu accessibles, difficilement identifiables et peu capables de mener à bien leur mission, ils ont d'autant moins l'intention de leur accorder leur confiance. Néanmoins, les analyses mettent en évidence un effet d'interaction significatif et négatif entre la légitimité pragmatique des associations de consommateurs et la vulnérabilité perçue des individus $(\beta=-$ 0,$08 ; p<0,05$ ) (Figure 3). En d'autres termes, alors que l'effet de la légitimité pragmatique sur l'intention de confiance est toujours positif, il se révèle d'autant plus fort que les individus ne se sentent pas vulnérables face aux acteurs marchands. Inversement, cet effet est d'autant plus faible que les consommateurs se sentent vulnérables. Cela signifie que plus les consommateurs se perçoivent comme vulnérables face aux acteurs marchands, moins la capacité perçue des associations de consommateurs à agir pour protéger leurs intérêts influence significativement leur intention de leur accorder leur confiance (comparativement aux individus qui se sentent peu vulnérables). Ce résultat est contraire à l'hypothèse H3b qui supposait que la vulnérabilité perçue des individus les porterait à avoir d'autant plus confiance dans les associations de consommateurs qu'ils les percevraient capables de mobiliser des ressources pour les protéger (H3b non validée).

\section{Tableau 4 et Figure 3 à insérer ici}


Effets de la légitimité morale et de la vulnérabilité perçue sur l'intention de faire confiance aux associations de consommateurs

Les effets du jugement de légitimité morale des associations de consommateurs, de la vulnérabilité perçue des individus et de leur interaction sur l'intention de leur faire confiance (H4) ont été analysés par le troisième modèle de régression (Modèle 3: $\mathrm{R}^{2}=42 \% ; F=$ 45,45***) (Tableau 5). Les résultats montrent une influence significative de la légitimité morale des associations de consommateurs sur l'intention des individus de leur accorder leur confiance $(\beta=0,25 ; p<0,01)$ (H4a validée). Ainsi, plus les consommateurs jugent que les associations de consommateurs poursuivent des objectifs légitimes pour la société, plus ils ont l'intention de leur accorder leur confiance. Ce résultat n'est pas modéré par l'effet de la vulnérabilité perçue des individus $(\beta=-0,02 ; p=0,48)$, autrement dit, cet effet n'est pas plus ou moins fort selon le niveau de vulnérabilité perçue des individus (Figure 4) (H4b non validée).

\section{Tableau 5 et Figure 4 à insérer ici}

\section{Discussion : implications, limites et voies de recherche}

Cette recherche avait pour objectif de tester l'effet explicatif de la légitimité des associations de consommateurs sur l'intention de leurs publics de leur faire confiance et d'identifier le rôle direct et modérateur de la vulnérabilité perçue des individus face aux acteurs marchands dans cette relation. Elle montre que les consommateurs ont d'autant plus l'intention d'accorder leur confiance aux associations de consommateurs qu'ils les perçoivent comme ayant toujours mené les mêmes missions (cognitivement légitimes), efficaces, accessibles et connues (pragmatiquement légitimes), ainsi qu'utiles sur le plan sociétal (moralement légitimes). Par ailleurs, les résultats montrent que l'intention de confiance est d'autant plus forte que les consommateurs se sentent vulnérables face aux acteurs marchands. Ces deux résultats conformes aux hypothèses prennent toutefois un autre relief lorsque l'effet modérateur de la vulnérabilité sur la relation entre la légitimité et l'intention de confiance est pris en compte. En effet, ce modérateur ne joue un rôle que dans le cas de la légitimité pragmatique. Et contrairement à ce qui était supposé dans l'hypothèse H3b, l'effet de la légitimité pragmatique est plus fort lorsque les individus ne se sentent pas vulnérables face aux acteurs marchands. Autrement dit, moins l'individu se perçoit comme fragile ou en position de faiblesse par rapport aux professionnels, plus l'efficacité, l'accessibilité et la notoriété perçues des associations de consommateurs ont une influence sur l'intention de leur faire confiance. Ce résultat contredit l'hypothèse qui découlait des travaux antérieurs (Roux, 2012 ; Roux, 
Chevalier et Nabec, 2015). Ceux-ci laissaient supposer, en effet, que l'efficacité, l'accessibilité et la notoriété des associations de consommateurs susciteraient plus de confiance chez des consommateurs vulnérables.

En revanche, les résultats de cette recherche ne montrent aucun effet d'interaction entre la légitimité cognitive et morale des associations de consommateurs et la vulnérabilité perçue des individus face aux acteurs marchands. Autrement dit, peu importe que la vulnérabilité perçue soit forte ou faible lorsque l'effet de la légitimité perçue sur l'intention de confiance porte sur le caractère institutionnel ou socialement désirable des associations. Il est donc fondamental que ces dernières travaillent à promouvoir leur expérience, leur histoire, leur impartialité, pour pouvoir convaincre les consommateurs dans leur ensemble, c'est-à-dire non seulement et prioritairement les publics qui se perçoivent en situation de vulnérabilité mais aussi ceux qui ressentent moins ce besoin de protection de leurs intérêts.

\section{Implications théoriques}

Ces résultats complètent les travaux existants sur la vulnérabilité ressentie des consommateurs (Baker, Gentry et Rittenburg, 2005) et la légitimité perçue des organisations (Capelli et Sabadie, 2005 ; Suchman, 1995). Ils éclairent la manière dont les associations doivent tenir compte des profils de leurs publics, notamment de leur vulnérabilité perçue. Les associations de consommateurs s'adressent, en effet, en majorité à des publics qui se perçoivent comme étant vulnérables face aux acteurs marchands. C'est précisément ce qui justifie leur existence et leur action auprès d'eux.

En premier lieu, les résultats de la recherche confirment que, dans son ensemble, la vulnérabilité perçue des individus influence bien l'intention de leur faire confiance. Ils enrichissent, dans un contexte différent, les études antérieures montrant que plus l'individu est vulnérable, plus il est enclin à accorder sa confiance, et plus les attentes qu'il peut avoir à l'égard du comportement d'autrui sont positives (Luhmann, 1988). En cela, cette recherche complète également les travaux sur l'intention de confiance dans les organisations, en soulignant l'intérêt théorique de la prise en compte de la vulnérabilité perçue des individus dans ce processus (Baker, Gentry et Rittenburg, 2005).

En second lieu, nos résultats montrent que la vulnérabilité modère l'influence de la légitimité pragmatique sur l'intention de confiance: cette dernière est d'autant plus forte que les individus ne se sentent pas particulièrement vulnérables face aux acteurs marchands. Ce résultat constitue un autre apport théorique : les personnes les moins vulnérables ont une plus grande intention de confiance dans les associations de consommateurs lorsqu'ils les 
considèrent comme efficaces, connues et faciles à contacter. Ce résultat suggère que les personnes les moins vulnérables sont davantage capables d'envisager les associations de consommateurs comme des ressources lorsque ces conditions sont réunies. A l'inverse, les individus les plus vulnérables, qui se sentent « dépourvus de contrôle », ont l'intention de faire confiance aux associations indépendamment de leur légitimité pragmatique. Ils ne se sentent peut-être pas capables de la juger. Ces résultats importants n'étaient pas encore apparus dans la littérature antérieure.

Enfin, si la relation entre légitimité et intention de soutenir une entreprise avait déjà été mise en évidence (Handelman et Arnold, 1999), le lien entre les trois dimensions du jugement de légitimité (Sabadie et Capelli, 2009 ; Suchman, 1995) et l'intention de confiance dans une association de consommateurs n'avait pas été mesuré jusqu'à présent. Cette recherche montre que la légitimité est un levier sur lequel il est possible d'agir pour susciter une plus grande confiance, y compris dans des organisations non marchandes, dans lesquelles la légitimité est aussi questionnée (Roux, Chevalier et Nabec, 2015 ; Vestergaard, 2014).

\section{Implications pour les associations de consommateurs}

En premier lieu, cette recherche souligne l'importance pour les associations de consommateurs d'envisager divers moyens permettant de renforcer la perception de leur légitimité. Les actions possibles sont complexes car elles dépendent du temps et des jeux institutionnels qui donnent plus ou moins de pouvoir au mouvement consommateur. Le rôle de l'État est, sur ce point, fondamental. Inversement, la réduction des financements publics est de nature à les fragiliser et à amenuiser encore davantage leurs maigres moyens de reconnaissance dans un paysage informationnel déjà largement encombré (Nabec et al., 2015).

Par ailleurs, il est essentiel que les associations de consommateurs puissent communiquer sur l'efficacité de leurs activités et sur les succès qu'elles remportent dans certaines affaires ou la défense de certains droits. Or, leurs actions de terrain quotidiennes, comme leur participation active au sein de multiples instances consultatives sont peu mises en avant. Une première recommandation viserait donc à mieux les valoriser pour renforcer la perception de leur légitimité pragmatique et donc la confiance qu'elles peuvent susciter. Moyennant de faibles investissements financiers, elles pourraient diffuser dans leurs différents canaux de communication (brochures, sites Internet...) les témoignages des consommateurs qui les ont sollicitées afin de faire connaître les résultats de ces actions. Comme le préconisait le rapport Laurent (2009) sur le monde consumériste, il serait également intéressant de réfléchir à la notion de mesure de la performance des associations. Les indicateurs restent toutefois à 
construire en gardant à l'esprit la souplesse que le monde associatif doit conserver pour répondre le plus efficacement possible à des réalités de terrain hétérogènes et complexes. Enfin, concernant la légitimité morale, si les associations sont réputées indépendantes des acteurs marchands, il est important que leurs publics en aient la certitude. Il est donc essentiel qu'ils ne doutent pas des objectifs qu'elles poursuivent en dissipant les suspicions de collusions entre fabricants et associations pour la réalisation des tests comparatifs (Roux, Chevalier et Nabec, 2015). Alors que l'UFC-Que Choisir développe désormais « La Note Que Choisir » pour permettre aux consommateurs d'identifier les produits les mieux classés dans ses tests comparatifs, le fait que les professionnels puissent acquérir le droit d'utiliser cette note moyennant paiement d'une licence ${ }^{6}$ peut, en dépit des précautions prises par l'association, être source d'amalgames dans un climat de scepticisme élevé (Darke, Ashworth et Ritchie, 2008).

En second lieu, cette recherche a permis de mettre en évidence l'importance de la vulnérabilité perçue face aux professionnels pour expliquer l'intention de confiance envers les associations de consommateurs. En complément du profil sociodémographique de leurs cibles, celles-ci pourraient chercher à mieux appréhender cette variable. En effet, il est important de tenir compte de cet état de vulnérabilité perçue, afin d'envisager des moyens d'action adaptés à ces profils. L'idée est que les associations arrivent à être perçues comme efficaces et suscitent donc une confiance parmi les publics les plus fragiles, qu'il convient de fait d'identifier.

\section{Limites et perspectives de recherche}

Cette recherche comporte un certain nombre de limites qui permettent d'envisager des voies de recherche futures. Tout d'abord, l'étude n'a porté que sur les associations de consommateurs agréées. Il serait intéressant de généraliser ces résultats à d'autres types d'organisations visant à défendre les consommateurs comme, par exemple, les associations non agréées dont les actions n'ont parfois pas moins d'efficacité que celles qui le sont. De même, la validité externe de la recherche pourrait être étendue, entre autres, aux collectifs de consommateurs organisés sur Internet, aux sites d'avocats qui cherchent à les assister, aux media TV et radio qui participent en direct à la résolution de certains litiges.

Deuxièmement, l'étude pourrait être prolongée en examinant la légitimité perçue de chaque association de consommateurs. Dans cette recherche, nous nous sommes intéressées à une

\footnotetext{
${ }^{6}$ Cette licence sert à couvrir les frais de gestion et de contrôle du bureau Véritas qui intervient comme organisme tiers entre l'UFC et le professionnel qui souhaite utiliser la « Note Que Choisir », le reliquat étant affecté par l'association à un fonds de dotation dédié à des campagnes d'information et d'éducation des consommateurs.
} 
perception globale, considérant les associations comme une institution chargée d'une mission commune. Un prolongement consisterait à comparer les différences de perception entre ces organisations afin de les aider individuellement à améliorer leur performance (Laurent, 2009). Troisièmement, une variable modératrice individuelle a été introduite dans cette recherche : la vulnérabilité perçue du consommateur face aux professionnels. Cette approche très générale gomme d'éventuelles variations de vulnérabilité selon les situations de consommation. Il conviendrait en conséquence de les explorer de manière différenciée. En effet, un consommateur peut se sentir vulnérable en tant qu'acheteur d'un contrat de téléphonie, mais moins lorsqu'il choisit un produit dans son domaine d'expertise, par exemple un article de sport. Il serait également intéressant de réfléchir à l'effet modérateur d'autres variables individuelles comme la propension à faire confiance aux autres (Rotter, 1967 ; 1971 ; 1980) ou à résister à la consommation (Roux, 2007 ; Banikéma et Roux, 2014). Le risque perçu, qui est un antécédent de la confiance (Benamour, 2000 ; Filser, 1998 ; Lewis et Weigert, 1985 ; Mitchell, 1999), pourrait être également une variable à considérer du fait que la confiance est liée à la perception de risques encourus (Benamour, 2000). Ceux-ci incluent par exemple le risque financier lié à une perte d'argent engendrée par les frais d'adhésion à une association de consommateurs (si l'action n'aboutit pas favorablement); le risque de perte de temps passé en démarches, notamment si la réparation n'est pas financièrement substantielle ; le risque psychologique découlant de la déception vis-à-vis de soi-même en cas d'échec ; ou encore le risque lié à l'anxiété que les individus peuvent ressentir dans des démarches longues dont l'issue est parfois hasardeuse. En quatrième lieu, et en dehors de ces variables individuelles, des facteurs situationnels peuvent expliquer l'intention de faire confiance à une association. Il serait pertinent de prendre en compte l'humeur au moment de l'étude, le moment de l'année ou le rôle de l'entourage. Ces résultats ouvrent donc un vaste champ de recherches à l'intersection des questions de légitimité, de confiance et de prise en compte de la vulnérabilité des individus dans le champ de la consommation. 


\section{Références}

Aiken L.S. et West S.G. (1991), Multiple regression: Testing and interpreting interactions.

Newbury Park: Sage.

Anderson JC et Gerbing DW (1988) Structural equation modeling in practice: a review and recommended two-step approach. Psychological Bulletin 103: 411-423.

Arnold S J, Handelman J et Tigert DJ (1996) Organizational legitimacy and retail store patronage. Journal of Business Research 35(3): 229-239.

Aurier P, Bénavent C et N'Goala G (2001) Validité discriminante et prédictive des composantes de la relation à la marque. Congrès de l'Association Française du Marketing, Deauville, 22-23 Mai.

Azar-Baud MJ (2016) La mise en ouvre des droits des consommateurs : ce que l'action de groupe va changer. In D Roux et L Nabec (eds) Protection des consommateurs. Les nouveaux enjeux du consumérisme. Cormelles-le-Royal: EMS, 193-204.

Baker SM, Gentry JW et Rittenburg TL (2005) Building understanding of the domain of consumer vulnerability. Journal of Macromarketing 25(2): 128-139.

Banikéma A et Roux D (2014) La propension à résister du consommateur : contribution a l'étude d'une disposition à s'opposer aux tentatives d'influence marchande. Recherche et Applications en Marketing 29(2): 34-59.

Bartikowsky B, Chandon JL et Müller B (2007) Mesurer la confiance des internautes dans les sites web marchands : adaptation de l'échelle de McKnight, Kacmar et Choudhury (2002). Working Paper, CERGAM: Université d'Aix-Marseille.

Benamour Y (2000) Confiance interpersonnelle et confiance institutionnelle dans la relation client-entreprise de service : une application au secteur bancaire français. Thèse de doctorat en sciences de gestion. Paris: Université Paris Dauphine.

Benedicktus RL, Brady MK, Darke PR et Voorhees CM (2010) Conveying Trustworthiness to Online Consumers: Reactions to Consensus, Physical Store Presence, Brand Familiarity, and Generalized Suspicion. Journal of Retailing 86(4): 310-323.

Bidault F et Jarillo JC (1995) La confiance dans les transactions économiques. In F Bidault, PY Gomez et G Marion (eds) Confiance, Entreprise et Société. Paris: Eska, 109-123.

Bihl-Willette L (1984) Une histoire du mouvement consommateur : mille ans de lutte. Paris: Aubier.

Bitektine A (2011) Towards a theory of social judgments of organizations: The case of legitimacy, reputation, and status. Academy of Management Review 36(1): 151-179. 
Bourdieu J et Pinto L (eds) (2013) Pauvre consommateur. Actes de la recherche en sciences sociales, 199. Paris: Seuil.

Bryce HJ (2007) The public's trust in nonprofit organisations. California Management Review 49(4): 112-131.

Buskirk RH et Rothe JT (1970) Consumerism. An interpretation. Journal of Marketing 34(4) $61-65$.

Cadario R et Parguel B (2014) Reconsidérer la discrétisation des variables quantitatives : vers une nouvelle analyse de modération dans la recherche expérimentale. Recherche et Applications en Marketing 29(4): 1-18.

Calais-Auloy J et Steinmetz F (1996) Droit de la consommation. Paris: Dalloz.

Capelli S et Sabadie W (2005) La légitimité d'une communication sociétale : le rôle de l'annonceur. Recherche et Applications en Marketing 20(4): 53-70.

Chaney D. et Ben Slimane K. (2014) La grille néo-institutionnelle au bénéfice de l'élargissement du marketing à des dimensions institutionnelles. Recherche et Applications en Marketing 17(2): 99-117.

Chatriot A (2004) Qui défend le consommateur ? Associations, institutions et politiques publiques en France (1972-2003). In A Chatriot, M-E Chessel et M Hilton (eds), Au nom du consommateur. Consommation et politique en Europe et aux États-Unis au XXe siècle. Paris: La Découverte, 165-181.

Chazal JP (2000) Vulnérabilité et droit : le développement de la vulnérabilité. Grenoble: Presses Universitaires de Grenoble.

Chessel M-E (2012) Consommateurs engagés à la Belle Époque. La Ligue sociale d'acheteurs. Paris: Presses de Sciences Po.

Chessel M-E (2016). Postface. Consommation : les «nouveautés » au regard de l'histoire. In D Roux et L Nabec (eds), Protection des consommateurs : les nouveaux enjeux du consumérisme. Cormelles-le-Royal: EMS, Collection Versus, 205-211.

Chevalier C, Nabec L et Roux D (2011) Les consommateurs face à l'information consumériste et à ses acteurs : représentations, attentes et enjeux. Actes du 27ème congrès de l'Association Française du Marketing, Bruxelles, 19-20 mai.

Chin WW et Newsted PR (1999) Structural equation analysis with small samples using partial least squares. In R Hoyle (ed), Statistical Strategies for Small Sample Research. London: Sage Publication, 307-341. 
Chouk I et Perrien J (2003) Les déterminants de la confiance du consommateur lors d'un achat sur un site marchand : proposition d'un cadre conceptuel préliminaire. Centre de recherche DMSP, cahier 318: 1-19.

Cissé-Depardon K et N'Goala G (2009) Les effets de la satisfaction, de la confiance et de l'engagement vis-à-vis d'une marque sur la participation des consommateurs à un boycott. Recherche et Applications en Marketing 24(1): 43-67.

Darke PR, Ashworth LTA et Ritchie RB (2008) Damage From Corrective Advertising: Causes and Cures. Journal of Marketing 72(6): 81-97.

Deutsch M (1962) Cooperation and trust: some theoretical notes. Nebraska Symposium on Motivation 10: 275-318.

Eichholtzer M (2010) Transparence, légitimité et confiance dans la gouvernance européenne. Mémoire de séminaire. Lyon: Institut d'Etudes Politiques de Lyon.

Fernandez V (2012) En quoi l'approche PLS est-elle une méthode à (re)découvrir pour les chercheurs en management ? Management 15(1): 101-123.

Filser M (1998) Confiance et comportement du consommateur. Economies et Sociétés, Sciences de Gestion Série S.G. 8-9: 279-294.

Fontaine L (2014) Le Marché. Histoire et usages d'une conquête sociale. Paris: Éditions Gallimard, NRF Essais.

Fornell C et Larker DF (1981) Evaluating structural equations models with unobservable variables and measurement error. Journal of Marketing Research 18(1): 39-50.

Gabaix X, Landier A et Thesmar D (2012) Protection du consommateur : Rationalité Limitée et Régulation. Paris: Rapport du Conseil d'Analyse Économique.

Gide C (1898/1931) Principes d'économie politique. Paris: Larose \& Forcel. http://classiques.uqac.ca/classiques/gide_charles/principes_economie_pol/principes_eco_po 1.html

Gorge H, Özçağlar-Toulouse N et Toussaint S (2015) Bien-être et well-being dans la consommation: une analyse comparative. Recherches et Applications en Marketing 30(2): $104-123$.

Guibert N (1999) La confiance en marketing: fondements et applications. Recherche et Applications en Marketing 14(1): 1-19.

Gurviez P et Korchia M (2002) Proposition d'une échelle de mesure unidimensionnelle de la confiance dans la marque. Recherche et Applications en Marketing 17(3): 41-61. 
Gustafsson A, Johnson MD et Roos I (2005) The effects of customer satisfaction, relationship commitment dimensions, and triggers on customer retention. Journal of Marketing 69: $210-218$.

Handelman JM et Arnold SJ (1999) The Role of Marketing Actions with a Social Dimension: Appeals to the Institutional Environment. Journal of Marketing 63(3): 33-48.

Hayes SC (2012) Humanistic psychology and contextual behavioural perspectives. Psychotherapy 49(4): 455-460.

Janz NK et Becker MH (1984) The health belief model: a decade later. Health Education Quaterly 11(1): 1-47.

Laurent D (2009) Mission relative au mouvement consumériste en France. Secrétariat d'Etat à l'industrie et à la consommation. Paris: La Documentation Française.

Lewis JD et Weigert A (1985) Trust as a Social Reality. Social Forces 63(4): 967-985.

Lichtlé MC et Plichon V (2008) Mieux comprendre la fidélité des consommateurs. Recherche et Applications en Marketing 24(4): 121-141.

Luhmann N (1988), Familiarity, confidence, trust: Problems and alternatives. In G Gambetta (ed), Trust. New York: Basil Blackwell.

Maillard-Wilhelm M-C (2014) Compréhension du fonctionnement de l'appel à la peur et du rôle médiateur de la réactance situationnelle en communication préventive de l'anorexie. Thèse de sciences de gestion, Grenoble: Université de Grenoble.

Mayer RC, Davis JH et Schoorman FD (1995) An Integrative Model of Organizational Trust. The Academy of Management Review 20(3): 709-734.

McKnight DH, Kacmar CJ et Choudhury V (2004) Dispositional Trust And Distrust Distinctions in Predicting High- and Low-Risk Internet Expert Advice Site Perception. EService Journal 3(2): 35-55.

Martin R (1987) Le consommateur abusif. Paris: Dalloz.

Meier O et Schier G (2008) Quelles théories et principes d'actions en matière de gouvernance des associations. Revue Management \& Avenir 6(20): 179-198.

Mitchell VW (1999) Consumer perceived risk: conceptualisations and models. European Journal of Marketing 33(1/2): 163-195.

Morgan RM et Hunt SD (1994) The commitment trust theory of relationship marketing. Journal of Marketing 58(3): 20-38.

Morgan F, Schuler DK et Stoltman JJ (1995) A framework for examining the legal status of vulnerable consumers. Journal of Public Policy \& Marketing 14(2): 267-277. 
Moorman C, Zaltman G et Deshpandé R (1992) Relationships between providers and users of market research: The dynamics of trust. Journal of Marketing Research 29(3): 314-328.

Nabec L, Chevalier C, Briat E et Roux D (2015) Légitimité et stratégies de légitimation des associations de consommateurs dans le paysage consumériste actuel; Décisions Marketing 78: 63-77.

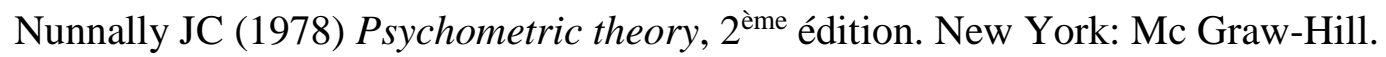

Pflieger G (2003) Consommateur, client, citoyen : l'usager dans les nouvelles régulations des services de réseaux. Les cas de l'eau, de l'électricité et des télécommunications en France. Thèse d'Urbanisme-Aménagement. Paris: Ecole des Ponts ParisTech.

Preacher KJ et Hayes AF (2004) SPSS and SAS procedures for estimating indirect effects in simple mediation models. Behavior Research Methods, Instruments, and Computers 36: $717-731$.

Preacher KJ et Hayes AF (2008) Asymptotic and resampling strategies for assessing and comparing indirect effects in multiple mediator models. Behavior Research Methods 40: 879-891.

Rindova VP, Pollock TG et Hayward MLA (2006) Celebrity firms: The social construction of market popularity. Academy of Management Review 31(1): 50-71.

Rossiter J (2002) The COARSE procedure for scale development in marketing. International Journal of Research in Marketing, 19: 305-335.

Rotter JB (1967) A new scale for the measurement of interpersonal trust. Journal of Personality 35(4): 651-665.

Rotter JB (1971) Generalized expectancies for interpersonal trust. American Psychologist 26: $443-452$.

Rotter JB (1980) Interpersonal trust, trustworthiness, and gullibility. American Psychologist 35: $1-7$.

Roux D (2007) Consumer resistance: proposal for an integrative framework, Recherche et Applications en Marketing (EBSCO English Edition) 22(4): 59-79.

Roux D (2012) Au-delà des réclamations : comprendre et gérer les litiges. Décisions Marketing 67: 49-62.

Roux D, Chevalier C et Nabec L (2015) Les parties prenantes consuméristes et l'impact de leur dynamique collective pour les entreprises. Décisions Marketing 77: 47-62.

Sabadie W et Capelli S (2009) Quelle légitimité à communiquer pour les candidats à l'élection présidentielle française : le cas de la communication sur l'environnement en 2007. Revue Française du Marketing 224(4/5): 21-34. 
Sinay-Cytermann A (1994) Protection ou surprotection du consommateur? La Semaine Juridique Edition Générale 48.

Sirieix L et Dubois PL (1999) Vers un modèle qualité-satisfaction intégrant la confiance ? Recherche et Applications en Marketing 14(3): 1-22.

Smith N et Cooper-Martin, E (1997) Ethics and Target Marketing: The Role of Product Harm and Consumer Vulnerability. Journal of Marketing 61(3): 1-20.

Smith JB et Barclay DW (1997). The Effects of Organizational Differences and Trust on the Effectiveness of Selling Partner Relationships. Journal of Marketing 61(1): 3-21.

Suchman MC (1995) Managing legitimacy: strategic and institutional approaches. Academy of Management Review 20(3): 571-610.

Tenenhaus M, Esposito Vinzi V, Chatelin Y et Lauro C (2005) PLS path modeling. Computational Statistics and Data Analysis 48: 159-205.

Thiétart RA (2003) Méthodes de recherche en management, 2ème édition. Paris:Dunod.

Vestergaard A (2014) Mediatized Humanitarianism: Trust and Legitimacy in the Age of Suspicion. Journal of Business Ethics 120(4): 509-525.

Webster FE (1973) Does business misunderstand consumerism? Harvard Business Review 51(5): 89-97.

Werle C, Balbo L, Ardito G et Vigne G (2010) Prévention de la surconsommation d'alcool au sein des grandes écoles françaises: l'effet boomerang des messages soulignant la vulnérabilité perçue. Journée Internationale du Marketing de la Santé, Lille, novembre. 